\title{
NuSTAR Observations of the Supernova Remnant RX J1713.7-3946
}

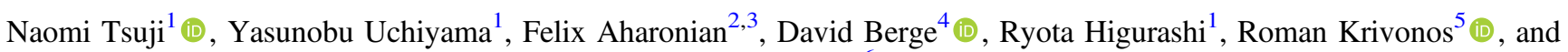 \\ Takaaki Tanaka ${ }^{6}$ (i) \\ ${ }^{1}$ Department of Physics, Rikkyo University, 3-34-1 Nishi Ikebukuro, Toshima-ku, Tokyo 171-8501, Japan; n.tsuji@ rikkyo.ac.jp, y.uchiyama@ rikkyo.ac.jp \\ ${ }^{2}$ Dublin Institute for Advanced Studies, 31 Fitzwilliam Place, Dublin 2, Ireland \\ ${ }^{3}$ Max-Planck-Institut für Kernphysik, P.O. Box 103980, D-69029 Heidelberg, Germany \\ ${ }^{4}$ DESY, Platanenallee 6, D-15738 Zeuthen, Germany \\ ${ }^{5}$ Space Research Institute of the Russian Academy of Sciences (IKI), Moscow, 117997, Russia \\ ${ }^{6}$ Department of Physics, Kyoto University, Kitashirakawa Oiwake-cho, Sakyo, Kyoto 606-8502, Japan \\ Received 2019 February 28; revised 2019 April 18; accepted 2019 April 18; published 2019 May 29
}

\begin{abstract}
The shock waves of supernova remnants (SNRs) are prominent candidates for the acceleration of the Galactic cosmic rays. SNR RX J1713.7-3946 is one well-studied particle accelerator in our Galaxy because of its strong nonthermal X-ray and gamma-ray radiation. We have performed Nuclear Spectroscopic Telescope Array (3-79 keV) observations of the northwest rim of RX J1713.7-3946, which is the brightest part in X-ray and has a shock speed of about $4000 \mathrm{~km} \mathrm{~s}^{-1}$. The spatially resolved X-ray emission from RX J1713.7-3946 is detected up to $20 \mathrm{keV}$ for the first time. The hard X-ray image in $10-20 \mathrm{keV}$ is broadly similar to the soft-band image in $3-10 \mathrm{keV}$. The typical spectrum is described by power-law model with exponential cutoff with the photon index $\Gamma=2.15$ and the cutoff energy $\varepsilon_{c}=18.8 \mathrm{keV}$. Using a synchrotron radiation model from accelerated electrons in the loss-limited case, the cutoff energy parameter ranges from $0.6-1.9 \mathrm{keV}$, varying from region to region. Combined with the previous measurement of the shock speed, the acceleration of electrons is close to the Bohmlimit regime in the outer edge, while the standard picture of accelerated particles limited by synchrotron radiation in SNR shock is not applicable in the inner edge and the filamentary structure.
\end{abstract}

Key words: acceleration of particles - ISM: individual objects (RX J1713.7-3946) - ISM: supernova remnants radiation mechanisms: non-thermal - X-rays: ISM

\section{Introduction}

Shell-type supernova remnant (SNR) RX J1713.7-3946 is well known for its strong nonthermal X-ray and gamma-ray emission, making it one of the best-studied particle acceleration sites (Tanaka et al. 2008; Abdo et al. 2011; H.E.S.S. Collaboration et al. 2018). The association between RX J1713.7-3946 and SN 393, one of the historical SNRs, has been discussed (Wang et al. 1997; Fesen et al. 2012). Recent measurements of the proper motions in the northwest (NW) and the southeast part of this SNR (Tsuji \& Uchiyama 2016; Acero et al. 2017) revealed that the forward shock speed is roughly $4000 \mathrm{~km} \mathrm{~s}^{-1}$. This suggests that RX J1713.7-3946 is indeed the remnant of SN 393 and kinematically young, implying it is still in the ejecta-dominated phase. Both the fast shock velocity and the early evolutional phase are consistent with the efficient acceleration of particles in the SNR.

The particle acceleration in RX J1713.7-3946 is likely in the most efficient regime. Zirakashvili \& Aharonian (2007) developed an analytical expression for the spectral energy distribution of electron around the SNR shock and the corresponding synchrotron radiation in the framework that the energy loss of the accelerated electrons is dominated by the synchrotron cooling (hereafter ZA07 model). Tanaka et al. (2008) reported that the broadband X-ray spectrum of the entire remnant of RX J1713.7-3946 with Suzaku (XIS and HXD PIN; $0.4-40 \mathrm{keV}$ ) is nicely described by the ZA07 model with the cutoff energy parameter $\left(\varepsilon_{0}\right)$ of $0.67 \mathrm{keV}$. Combined with the shock speed, they reported that the acceleration of electrons is in the regime close to the Bohm limit, where the so-called gyro factor is close to unity.
The Nuclear Spectroscopic Telescope Array (NuSTAR; Harrison et al. 2013) enables us to perform the first observation of the spatially resolved hard X-ray above $10 \mathrm{keV}$. The nonthermal (synchrotron) X-ray has been detected with NuSTAR from some young Galactic SNRs: e.g., Cassiopeia A (Grefenstette et al. 2015; Sato et al. 2018), Tycho's SNR (Lopez et al. 2015), G1.9+0.3 (Zoglauer et al. 2015; Aharonian et al. 2017), and SN 1006 ( $\mathrm{Li}$ et al. 2018). The spatially resolved spectral analysis was performed for these SNRs. In Cassiopeia A, Tycho's SNR, and SN 1006, the spectral properties are significantly different from region to region, suggesting that the efficiency of acceleration depends on the site. Although G1.9+0.3 shows uniform spectra across the remnant (Zoglauer et al. 2015), the acceleration of electrons is about one order of magnitude slower than the Bohm limit, as reported in Aharonian et al. (2017).

Our NUSTAR observations of RX J1713.7-3946 are the third example of the detections of the spatially resolved nonthermal hard X-ray emissions with NUSTAR from the synchrotron-dominated SNRs, following G1.9+0.3 and SN 1006. The hard X-ray emission from RX J1713.7-3946 was detected with INTEGRAL/IBIS in 17-60 keV (Krivonos et al. 2007a) and Suzaku/HXD PIN in 12-40 keV (Tanaka et al. 2008). However, the angular resolution of these X-ray instruments is limited: INTEGRAL/IBIS is a coded-mask telescope and Suzaku/HXD is a nonimaging, collimated instrument with the detector units of silicon PIN diodes and GSO scintillators. We investigate the spatial distribution of the hard X-ray on arcmin scale using NUSTAR, which consists of hard X-ray telescopes and detectors.

In this paper, we present the results of our spatially resolved hard X-ray observations obtained with NuSTAR from the NW 
Table 1

Log of NUSTAR Observations

\begin{tabular}{|c|c|c|c|c|c|c|c|}
\hline & \multirow{2}{*}{ ObsID } & \multirow{2}{*}{$\begin{array}{c}\text { Start Date } \\
\text { (yyyy mm dd) }\end{array}$} & \multicolumn{2}{|c|}{ Pointing Position } & \multirow{2}{*}{$\begin{array}{l}\text { Positional Angle } \\
\text { (degree) }\end{array}$} & \multirow{2}{*}{$\begin{array}{l}\text { Exposure } \\
\text { (ks) }\end{array}$} & \multirow{2}{*}{$\begin{array}{c}\text { Effective Time }{ }^{\mathrm{a}} \\
(\mathrm{ks})\end{array}$} \\
\hline & & & $(\alpha, \delta)^{\mathrm{b}}$ & $(l, b)^{\mathrm{c}}$ & & & \\
\hline P1 & 40111001002 & 2015 Sep 27 & $257.86,-39.52$ & $347.29,+0.001$ & 343.3 & 50 & 43 \\
\hline $\mathrm{P} 2$ & 40111002002 & 2016 Mar 30 & $257.93,-39.58$ & $347.28,-0.077$ & 165.6 & 57 & 49 \\
\hline $\mathrm{T} \mathrm{CrB}$ & 30101046002 & 2015 Sep 23 & $239.85,+25.90$ & $42.34,+48.18$ & 319.5 & 80 & 63 \\
\hline
\end{tabular}

Notes.

${ }^{\mathrm{a}}$ After screening with SAAMODE $=$ STRICT and TENTACLE $=$ YES cut.

${ }^{b}$ R.A. and decl. in units of degrees.

${ }^{c}$ Galactic longitude and latitude in units of degrees.

rim of RX J1713.7-3946. Section 2 summarizes the NuSTAR observations. The imaging and the spectral analysis are described in Section 3. We discuss the efficiency of particle acceleration in several arcmin-scale regions in the NW in Section 4. The conclusions are presented in Section 5.

\section{Observation}

With the NUSTAR satellite (Harrison et al. 2013), we have performed observations of the NW region of RX J1713.7 -3946 twice, in 2015 September (hereafter P1) and in 2016 March (P2), with exposure times of 50 and $57 \mathrm{ks}$, respectively (Table 1). These data are taken with two focal plane modules, named FPMA and FPMB, on board the NuSTAR satellite, coaligned with the corresponding optics modules. The energy range of both detectors is $3-79 \mathrm{keV}$.

All data are calibrated and screened by using nupipeline of NuSTAR Data Analysis Software (NuSTARDAS version 1.4.1 with CALDB version 20180814), included in HEAsoft version 6.19. For the data screening, we use the strictest mode $($ SAAMODE $=$ STRICT and TENTACLE $=$ YES cut $)$. This process reduces the effective observation time to 43 and $49 \mathrm{ks}$ for P1 and P2, respectively (Table 1).

\section{Analysis and Results}

\subsection{Detailed Analysis of NuSTAR}

NUSTAR has side aperture contamination. Due to the separation between the telescope modules and the detectors and its openness to the sky window, the X-ray emissions from the outside of the field of view (FOV) can be detected directly in the FPMs without passing through the optic modules. They are referred to as stray lights. They come from the region within radius of 2.5 from the center of the FOV. The stray lights are caused by bright X-ray sources located in the vicinity of the target point, Cosmic X-ray Background (CXB), and Galactic Ridge X-ray Emission (GRXE; Revnivtsev et al. 2006; Krivonos et al. 2007b; Yuasa et al. 2012) when pointing the Galactic plane. This causes complex distributions of the background.

Since RX J1713.7-3946 is located on the Galactic plane, there are some bright $\mathrm{X}$-ray sources as possible stray-light contaminators. For example, the stray light from HMXB $4 \mathrm{U}$ 1700-377 dominates in all X-ray energy bands except for P1 observation with FPMA. In addition, there exist stray-light contaminations from GX 349+2, 4U 1708-40 in P1-FPMA, and $4 \mathrm{U} 1702-429$ in P1-FPMA and P2-FPMB. Figure 1 illustrates the regions contaminated by the stray lights from these X-ray sources. We exclude these contaminated regions for the following imaging and spectral analysis.

Another component to cause stray-light contaminations is CXB. In addition to the focused CXB emission passing through the optics, CXB coming from the outside of the FOV without passing through the telescopes are detected nonuniformly in the FPMs. It should be noted that we need to take these nonuniform distributions into account because RX J1713.7 -3946 is extended across most of the FOV. In order to deal with this issue, we use the toolkit named "nuskybgd"7 (Wik et al. 2014).

Thanks to nuskybgd, we can construct the background for any region in which we are interested. The general usage of nuskybgd is as follows. First we extract the spectra of "sourcefree" regions outside the SNR, where any stray lights from $\mathrm{X}$-ray sources should not be contained. In nuskybgd, the model consists of the following four components: (1) "aCXB" to describe the stray-light CXB through the aperture, (2) "fCXB" to describe the focused CXB, (3) "Inst" to describe instrument line emissions and reflected solar X-rays, and (4) "Intn" to describe instrument Compton scattered continuum emissions. Using nuskybgd_fitab included in nuskybgd, we can fit the background spectrum by the model above. Based on the best-fit parameters, the background image and the background spectrum for an arbitrary region in the FOV are, respectively, produced by using nuskybgd_image and nuskybgd_spec in nuskybgd.

GRXE is not negligible because RX J1713.7-3946 is located on the Galactic plane. In this paper, we use the "apec" model in XSPEC with the electron temperature $(k T)$ of $8 \mathrm{keV}$ for GRXE. Indeed, off-set observations near RX J1713.7 -3946 with Suzaku showed that CXB and GRXE, which is described by apec with $k T=7.4-8.8 \mathrm{keV}$, are dominant above $3 \mathrm{keV}$ (Katsuda et al. 2015). Similar to CXB, GRXE has also a focused component ("fGRXE") and an unfocused component ("aGRXE"). We assume fGRXE is uniform in the vicinity of RX J1713.7-3946, and fix the normalization to the value derived from Suzaku observations in Katsuda et al. (2015). The distribution of aGRXE, however, is not well understood yet. Unlike aCXB coming from the flat sky, modeling aGRXE distribution on the detectors is a difficult task, because it mainly comes from the nonuniform Galactic bulge. Here we assume that aGRXE is simply uniform on the focal plane modules, and consider systematic uncertainties associated with this approximation in Section 3.3.

\footnotetext{
https://github.com/NuSTAR/nuskybgd
} 

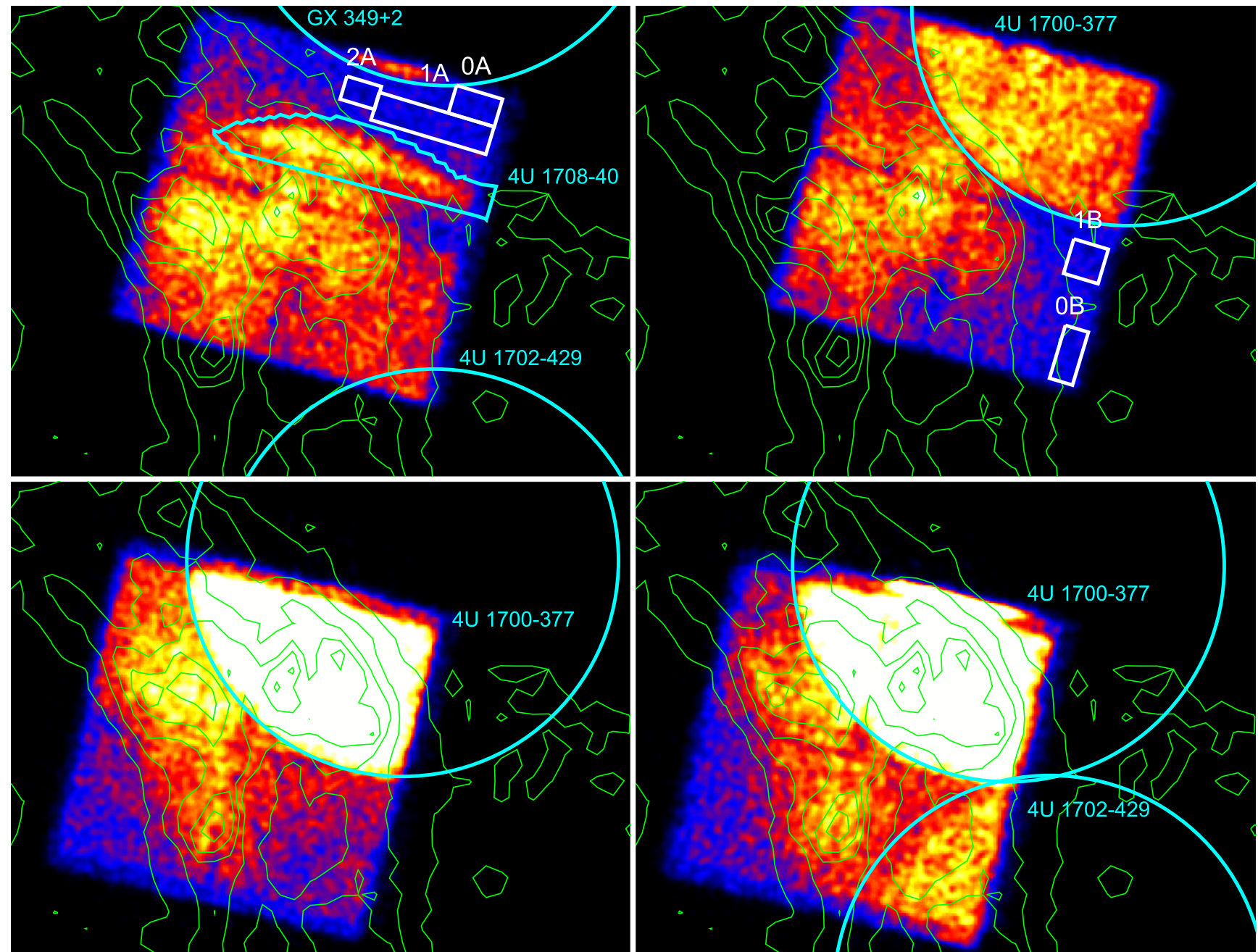

bkg OA
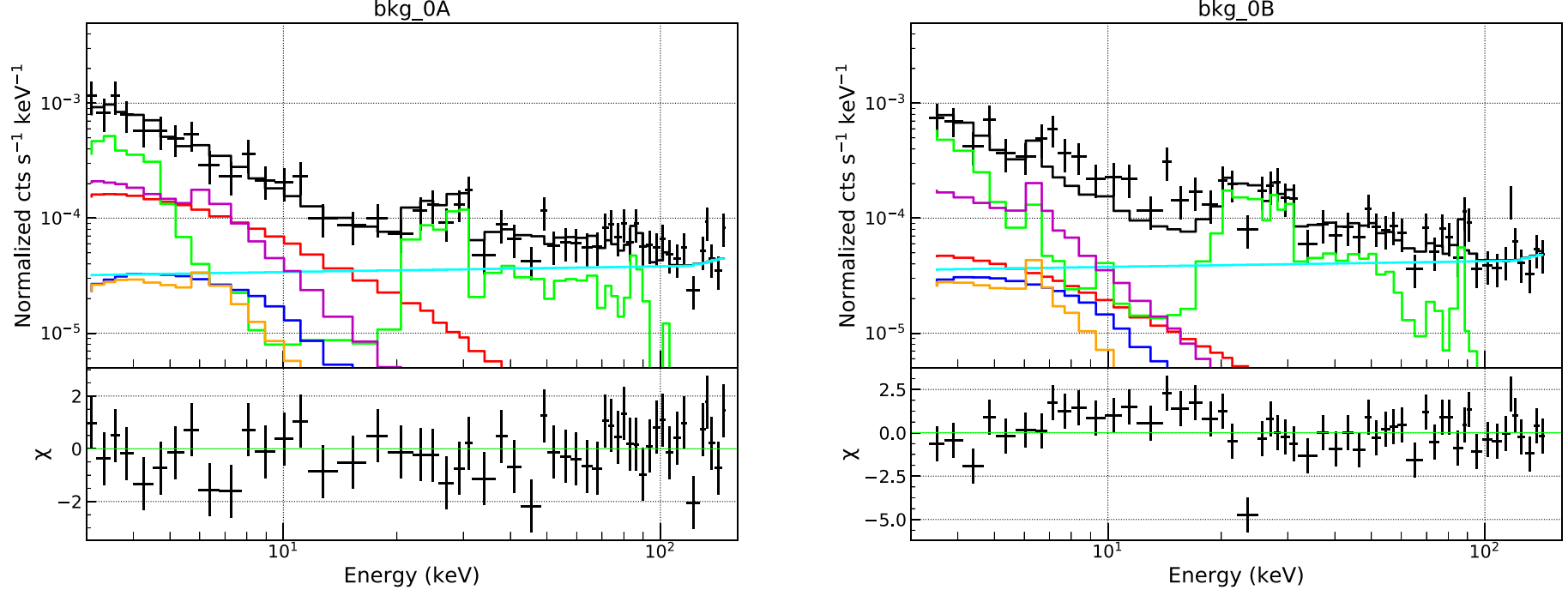

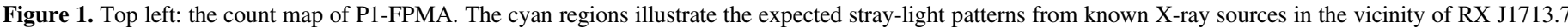

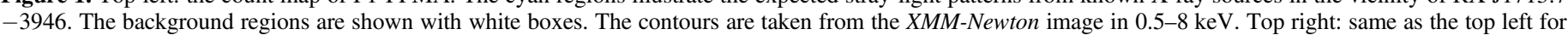

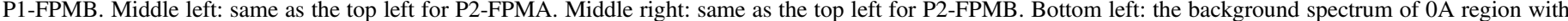

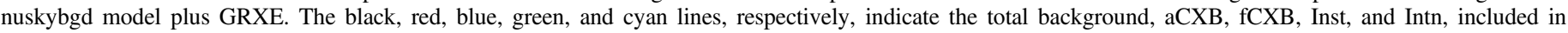
nuskybgd. The magenta and orange lines are aGRXE and fGRXE, respectively. Bottom right: same as the bottom left for the spectrum of 0B.

Our background estimation of RX J1713.7-3946 with NuSTAR is as follows. We extract the background spectra from the regions referred to $0 \mathrm{~A}-2 \mathrm{~A}$ in FPMA and $0 \mathrm{~B}-1 \mathrm{~B}$ in FPMB, shown in Figure 1. To estimate the background of FPMA, the spectra of $0 \mathrm{~A}-2 \mathrm{~A}$ are jointly fitted, tying the normalizations between them based on the relative brightnesses expected from the known nonuniform distribution. The spectra of $0 \mathrm{~B}-1 \mathrm{~B}$ are also jointly fitted to estimate the background of FPMB. 
Dividing the background region into a few pieces, such as $0 \mathrm{~A}$, 1A, and 2A, helps us to better estimate the background, as recommended in Wik et al. (2014). The background model consists of aCXB, fCXB, Inst, Intn, aGRXE, and fGRXE. We use nuskybgd for $\mathrm{CXB}$ and the instrumental components, while we add GRXE manually because the modeling of GRXE is not yet developed in nuskybgd. When fitting the background spectra, the normalizations of $\mathrm{CCXB}$ and $\mathrm{aCXB}$ are fixed to the following values. The normalization of fCXB is fixed to the value defined in the literature (Boldt 1987). Since CXB is uniform over the sky, the normalization of aCXB is derived from a high-latitude NUSTAR observation, where no GRXE is contained. The observation of a symbiotic star $\mathrm{T} \mathrm{CrB}$ with the latitude of $48^{\circ} .18$ is selected for this purpose (Table 1). We fit the background spectra of $\mathrm{T} \mathrm{CrB}$, which are extracted from the same detector region as that of RX J1713.7-3946, using nuskybgd in order to get the level of aCXB. The obtained normalization is then used for the normalization of $\mathrm{aCXB}$ for the observations of RX J1713.7-3946.

The spectra of $0 \mathrm{~A}$ and $\mathrm{OB}$ are shown with all the components of the background model in Figure 1. The background spectra are well-fitted by our background model. It is notable that the background spectrum of $\mathrm{OB}$ is extracted from the region where $\mathrm{aCXB}$ is weakest in FPMB, making the component of $\mathrm{aCXB}$ lower. The free parameters in low energy band and high energy band are, respectively, the normalization of aGRXE and the instrument emissions. The normalization of aGRXE is roughly obtained to be $\int n_{\mathrm{e}} n_{\mathrm{H}} d \ell=7 \times 10^{19} \mathrm{~cm}^{-5}$ for both FPMA and FPMB, based on the assumption of the distance of $1 \mathrm{kpc}$ (Fukui et al. 2003).

In order to construct the background spectrum for the region of interest, we use nuskybgd_spec in nuskybgd for aCXB, fCXB, Inst, and Intn components. It takes into account the nonuniform distribution of the background, i.e., the background normalization for the region of interest is weighted by the nonuniform distribution on the detectors. On the other hand, for fGRXE and aGRXE we simply rescale the normalization by the size of the source region, assuming the flat GRXE distribution across the FOV. We discuss the validity of this assumption in Section 3.3.

For P2 epoch, the background is estimated based on the bestfit parameters of the background of P1 observation. Since the emission of RX J1713.7-3946 is extended across the entire FOV of $\mathrm{P} 2$, there is no region to extract for the background estimation. We assume that the background with NuSTAR is approximately stable over the period of these two observations, P1 (2015 September) and P2 (2016 March). The background of an arbitrary region in $\mathrm{P} 2$ observation is generated in the same procedure as $\mathrm{P} 1$, as mentioned above.

\subsection{Image}

In Figure 2, we present the images in energy bands of $3-10 \mathrm{keV}$ and $10-20 \mathrm{keV}$. These are background-subtracted and exposure-corrected images, where the background images are constructed using nuskybgd and the exposure maps are generated by nuexpomap in NuSTARDAS with no vignetting correction, after removing the regions contaminated by the stray lights from X-ray sources. We note that GRXE is included in these images, while the other background, $\mathrm{CXB}$ and the instrument background, are subtracted. The observations of the two epochs and the two FPMs are combined. The
$3-10 \mathrm{keV}$ image is roughly consistent with the soft X-rays in $0.5-8 \mathrm{keV}$ taken by XMM-Newton shown with the contour.

We find an interesting feature, which is referred to as $\mathrm{HXC}$ (Hard X-ray Component), at the position of the green ellipse that is centered at $\left(\alpha_{\mathrm{J} 2000}, \quad \delta_{\mathrm{J} 2000}\right)=\left(17.11^{\mathrm{m}} .8 . " 68\right.$, $-39.37^{\mathrm{m}} .7$ !' 23 ) in the $10-20 \mathrm{keV}$ image, while the other part of the hard X-ray image is roughly in agreement with the soft $\mathrm{X}$-rays. This region is faint in the lower X-ray band, making its spectrum extremely hard $(\Gamma \sim 1)$. It should be noted that the region around $\mathrm{HXC}$ is not contaminated by any stray lights from X-ray sources. It remains as a future work to confirm the presence of HXC and interpret the physical meaning.

\subsection{Spectrum}

NuSTAR, for the first time, allows us to perform spatially resolved spectroscopy in the hard X-ray band above $\sim 10 \mathrm{keV}$. We investigate the hard X-ray spectral distribution for different regions in the NW of RX J1713.7-3946. All the spectra are produced using nuproducts in NuSTARDAS with parameter "extended" set to "yes." All the background spectra are generated by nuskybgd and adding GRXE, as described in Section 3.1. The spectral fitting is performed with XSPEC version 12.9.0.

The energy range of $N U S T A R$ spectra for the spectral analysis is set to be $3-20 \mathrm{keV}$ due to the poor statistics above $20 \mathrm{keV}$, caused by stronger instrumental background. Combining the NuSTAR spectra with the $0.5-7 \mathrm{keV}$ spectra obtained with Chandra, we perform Chandra $+N u S T A R$ joint fitting. For Chandra data, we use six observations of RX J1713.7-3946 NW with ObsID of 736, 6370, 10090, 10091, 10092, and 12671. The data are processed using CALDB version 4.7.6 in CIAO version 4.9. All the spectra of different epochs with Chandra are combined using addascaspec in HEAsoft.

The spectral fitting is performed for the integrated region (large box) and spatially resolved arcmin-scale regions (small box (a)-(e)), as shown in Figure 3. We use two conventional models, which are the power-law model and the power law with exponential cutoff model, as well as the ZA07 model. Zirakashvili \& Aharonian (2007) obtained the analytical expressions of the synchrotron radiation spectrum from accelerated electrons in the framework that assumes electrons accelerated via DSA are cooled by synchrotron emissions. The analytical solution of the electron spectrum and the synchrotron-photon spectrum in the downstream region are, respectively,

$$
\begin{aligned}
\frac{d N_{e}}{d E} \propto\left(\frac{E}{E_{0}}\right)^{-3}\left[\left\{1+0.523\left(\frac{E}{E_{0}}\right)^{\frac{9}{4}}\right\}^{2}\right. & \left.-0.0636\left(\frac{E}{E_{0}}\right)^{2}\left\{1+1.7\left(\frac{E}{E_{0}}\right)^{3}\right\}^{\frac{5}{6}}\right] \exp \left[-\left(\frac{E}{E_{0}}\right)^{2}\right], \\
& \frac{d N}{d \varepsilon} \propto\left(\frac{\varepsilon}{\varepsilon_{0}}\right)^{-2}\left[1+0.38\left(\frac{\varepsilon}{\varepsilon_{0}}\right)^{\frac{1}{2}}\right]^{\frac{11}{4}} \exp \left[-\left(\frac{\varepsilon}{\varepsilon_{0}}\right)^{\frac{1}{2}}\right],
\end{aligned}
$$

which are the same as Equations (28) and (37) in Zirakashvili $\&$ Aharonian (2007). Here $E_{0}$ and $\varepsilon_{0}$ are the cutoff energy parameters of the electron and the synchrotron X-ray, respectively. Note that the cutoff energy $\left(\varepsilon_{c}\right)$ in the cutoff 

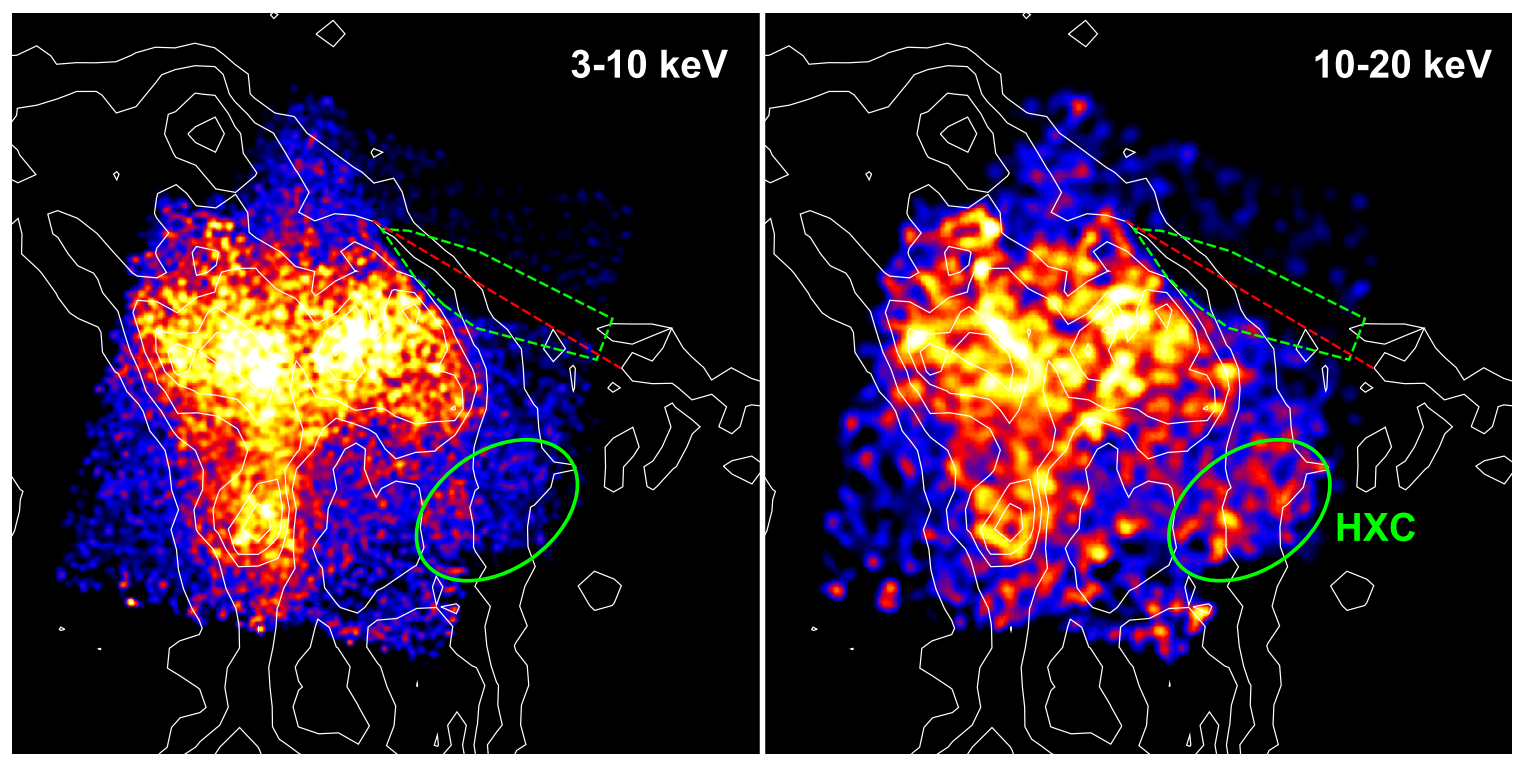

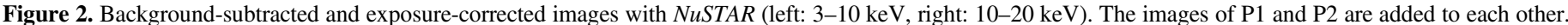

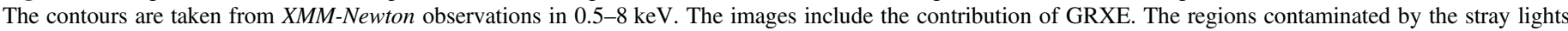

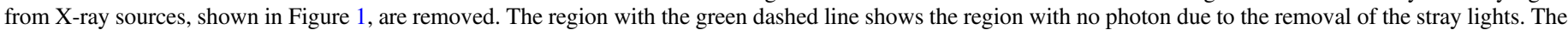
position of $\mathrm{HXC}$ is illustrated with the green ellipse.

power-law model is about one order of magnitude higher than $\varepsilon_{0}$ in the ZA07 model. This is because $\varepsilon_{c}$ gives $e^{-1}$ deviation from the power law, while $\varepsilon_{0}$ does not due to the additional function to the cutoff power law (Equation (2)). The interstellar absorption is taken into account for all the models using the "TBabs" model in XSPEC.

We take the uncertainty of the background spectrum into consideration as a systematic error. When fitting the background spectra with nuskybgd_fitab in nuskybgd, the bestfit parameters have about $10 \%$ error. Therefore, we check how much the source spectrum from the region of interest depends on the choice of the background spectrum, by changing the normalization. If we change the normalization of the total background spectrum by $10 \%$, the source spectrum changes by $10 \%$. This uncertainty is used as the systematic error in the following. It should be noted that the uncertainty of the aGRXE component could be much larger than the statistical error because we simply assume the uniform distribution of aGRXE in Section 3.1. If we change only the normalization of the aGRXE spectrum by a factor of 3 , the source spectrum changes by $10 \%$. Thus our assumption on the uniform aGRXE does not largely affect the source spectrum as long as aGRXE is nonuniformly distributed by a factor of $\leqslant 3$. We note that in fact CXB is expected to fluctuate by $\sim 20 \%$ in the FOV of NuSTAR (Moretti et al. 2009). Changing the normalization of aCXB, we also checked that this uncertainty does not have a large effect on the source spectrum, i.e., the cutoff energy changes by $\sim 15 \%$, and the other parameters remain the same within $\sim 2 \%$ for $20 \%$ fluctuation of aCXB.

Figure 4 (left) shows the spectrum of the large box. The angular size of the box is $5.7 \times 4.8$, which corresponds to $1.7 \times 1.4 \mathrm{pc}^{2}$ assuming the distance is $1 \mathrm{kpc}$ (Fukui et al. 2003). This large box represents the typical spectrum in the NW of RX J1713.7-3946. The best-fit parameters are listed in Table 2. Using the power-law model, the photon index of the NuSTAR spectrum in $3-20 \mathrm{keV}$ is $2.55 \pm 0.04 \pm 0.02$, which is larger than that of the Chandra spectrum in $0.5-7 \mathrm{keV}$ with
$\Gamma=2.32 \pm 0.02$. Here the first and second errors indicate the statistic and the systematic error, respectively. For the broadband energy spectrum (Chandra $+N u S T A R$ ), our analysis excludes the pure power-law model and requires the cutoff in the spectrum. The cutoff shape is described with the photon index $\Gamma=2.15 \pm 0.04 \pm 0.02$ and $\varepsilon_{c}=18.8_{-3.0}^{+4.2}{ }_{-2.0}^{+2.6} \mathrm{keV}$ for the simple cutoff power-law model, and $\varepsilon_{0}=1.14 \pm 0.06 \pm 0.02 \mathrm{keV}$ for the ZA07. Note that the systematic error is less than the statistical error. The cutoff power-law model seems to be slightly favored, as inferred from the chi-squared value. However, we should be cautious about the coupling of the cutoff energy and the photon index. Both $\varepsilon_{c}$ and $\Gamma$ are higher than the values reported in Tanaka et al. (2008). Furthermore, the best-fit value of the photon index $(\Gamma=2.15)$ is larger than 2 , which is expected for the synchrotron X-ray spectrum radiated from the electron, which the acceleration is limited by the synchrotron cooling. If we fix $\Gamma$ to the theoretically predicted value of 2 , the chi-squared value becomes much larger. ZA07 model gives a better fit than the CPL model with $\Gamma=2$.

We report the first result of arcmin-scale spectral distribution in the hard X-ray band of the NW shell of RX J1713.7-3946. Six small regions, boxes (a)-(f), are selected for the following reasons. Boxes (a)-(f) include clearly edge-like and filamentlike structures. We have previously measured the proper motion velocities in these structures (Tsuji \& Uchiyama 2016) and found that the speeds are significantly different from region to region. To test the relation between the shock speed and the cutoff energy, we define boxes (a)-(f), which correspond to the regions used in Tsuji \& Uchiyama (2016). The size of each box is $1.5 \times 2^{\prime}\left(0.44 \times 0.58 \mathrm{pc}^{2}\right)$ except for box (f) with the size of $3^{\prime} \times 4^{\prime}\left(0.88 \times 1.16 \mathrm{pc}^{2}\right)$. The spectrum of box (a) is shown in Figure 4 and the best-fit parameters are listed in Table 2.

Figure 5 demonstrates the relation between the shock speed obtained by Tsuji \& Uchiyama (2016) and the cutoff energy parameter derived from the spectral fitting with the ZA07 model for each small box. The shock velocities are assumed to be the same as the proper motion velocities: 


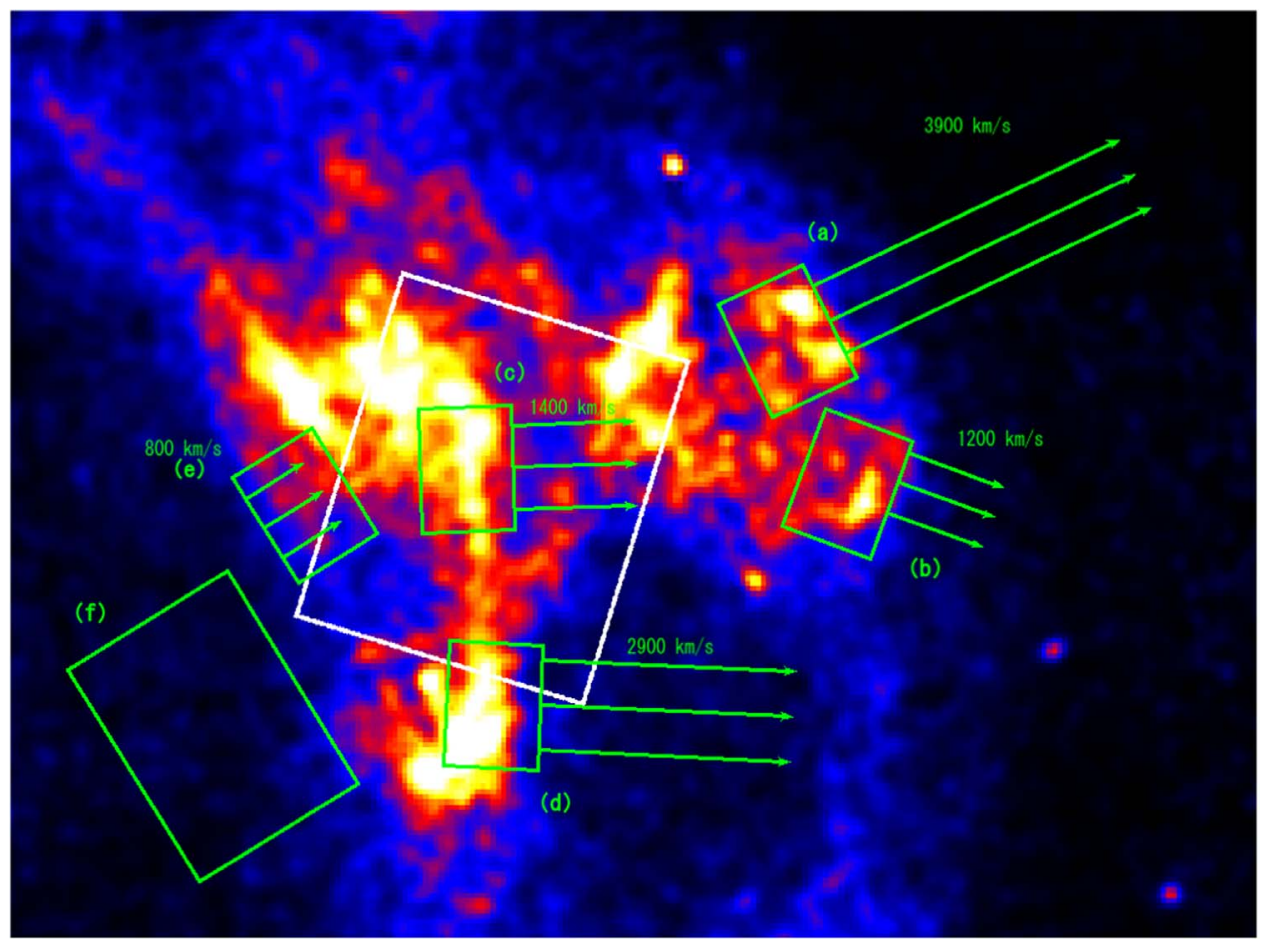

Figure 3. Locations of the large box and the small box (a)-(f), shown with the white box and the green boxes, respectively. The image is $0.5-8$ keV with $X M M$ Newton. The color is shown in squared scale. The length of an arrow indicates the shock speed measured by Chandra (Tsuji \& Uchiyama 2016).

$3900 \pm 300 \mathrm{~km} \mathrm{~s}^{-1}$ at box (a), $1200 \pm 300 \mathrm{~km} \mathrm{~s}^{-1}$ at box (b), $1400 \pm 200 \mathrm{~km} \mathrm{~s}^{-1}$ at box (c), $2900 \pm 200 \mathrm{~km} \mathrm{~s}^{-1}$ at box (d), and $800 \pm 300 \mathrm{~km} \mathrm{~s}^{-1}$ at box (e) and box (f), assuming the distance is $1 \mathrm{kpc}$ (Fukui et al. 2003). The measured speeds contain the uncertainties of being projected onto the line of sight. However, these uncertainties can be small because boxes (a)-(f) are located in the outer regions of the shell and the radial component is expected to be dominant in these regions. The projection-corrected speeds, plotted with the open markers in Figure 5, are inferred from taking into account the projection effects, i.e., the line-of-sight velocity components, assuming the spherical shell expansion.

\section{Discussion}

The cutoff energy in the synchrotron spectrum may contain key information about the mechanism responsible for acceleration of the parent particles. Zirakashvili \& Aharonian (2007) obtained the following relation between the shock speed and the cutoff energy parameter:

$$
\varepsilon_{0}=1.55\left(\frac{v_{\mathrm{sh}}}{3900 \mathrm{~km} \mathrm{~s}^{-1}}\right)^{2} \eta^{-1} \mathrm{keV},
$$

where $\eta \geqslant 1$ is the parameter to indicate the deviation from the Bohm limit, the so-called gyro factor. The $\eta=1$ case, known as the Bohm limit, is the most efficient acceleration. The theoretical relation, Equation (3), is shown by the green line in Figure 5.

We assume the ratio of the upstream magnetic field to the downstream magnetic field, $\kappa=B_{\text {up }} / B_{\text {down }}$, is $\sqrt{1 / 11}$. This indicates an enhancement of random isotropic magnetic field due to the standard shock compression with ratio of $\sigma=4$. In the case of the equal value of the magnetic field upstream and downstream, $\kappa=1$, Equations (1)-(3) are slightly modified
(Zirakashvili \& Aharonian 2007). Using the models for the $\kappa=1$ case, we also perform the same spectral analysis to the broadband X-ray observations with Chandra and NuSTAR. The observed $\varepsilon_{0}$ in the $\kappa=1$ case is smaller than that in the $\kappa=\sqrt{1 / 11}$ case by a factor of $0.6-0.7$, while the theoretically predicted $\varepsilon_{0}$ is smaller by a factor of 0.6. This results in a similar trend between the shock velocity and the cutoff energy parameter and the case of $\kappa=\sqrt{1 / 11}$ illustrated in Figure 5, which indicates that boxes (a) and (d) are roughly consistent with the theoretical curve, and the rest of the boxes are not.

As shown in Figure 5, the $v_{\mathrm{sh}}-\varepsilon_{0}$ relation of box (a) and box (d) can be explained by the theoretical relation with $\eta \sim 1$. TeVscale electrons are accelerated at the maximum rate (Bohm limit) in these outermost regions that are likely just behind the forward shock. Note that box (d), which seems to be located inside the shell, is possibly indicative of the projected forward shock (box (a)) because the projection-corrected speed is compatible with that of box (a).

On the other hand, the regions with the lower speed (boxes (b), (c), (e), and (f)) do not match the theoretical prediction. This suggests that the present framework, which we assume the synchrotron radiation from electrons accelerated at SNR shock through the standard DSA mechanism and limited by the synchrotron loss, is not applicable in these cases. The inner filament and/or edge at boxes (c), (e), and (f) may represent locally enhanced magnetic fields rather than the acceleration sites. Although box (b) exists at the outermost edge, its slow speed and its nonradial direction imply that the shock is decelerated and distorted due to interacting with the molecular clouds (Fukui et al. 2003, 2012; Sano et al. 2015). Therefore, box (b) might not be the acceleration site.

The magnetic field amplifications have been confirmed in the filaments and the small knot-like structures in the previous work of some young SNRs. Estimated from the width of the 

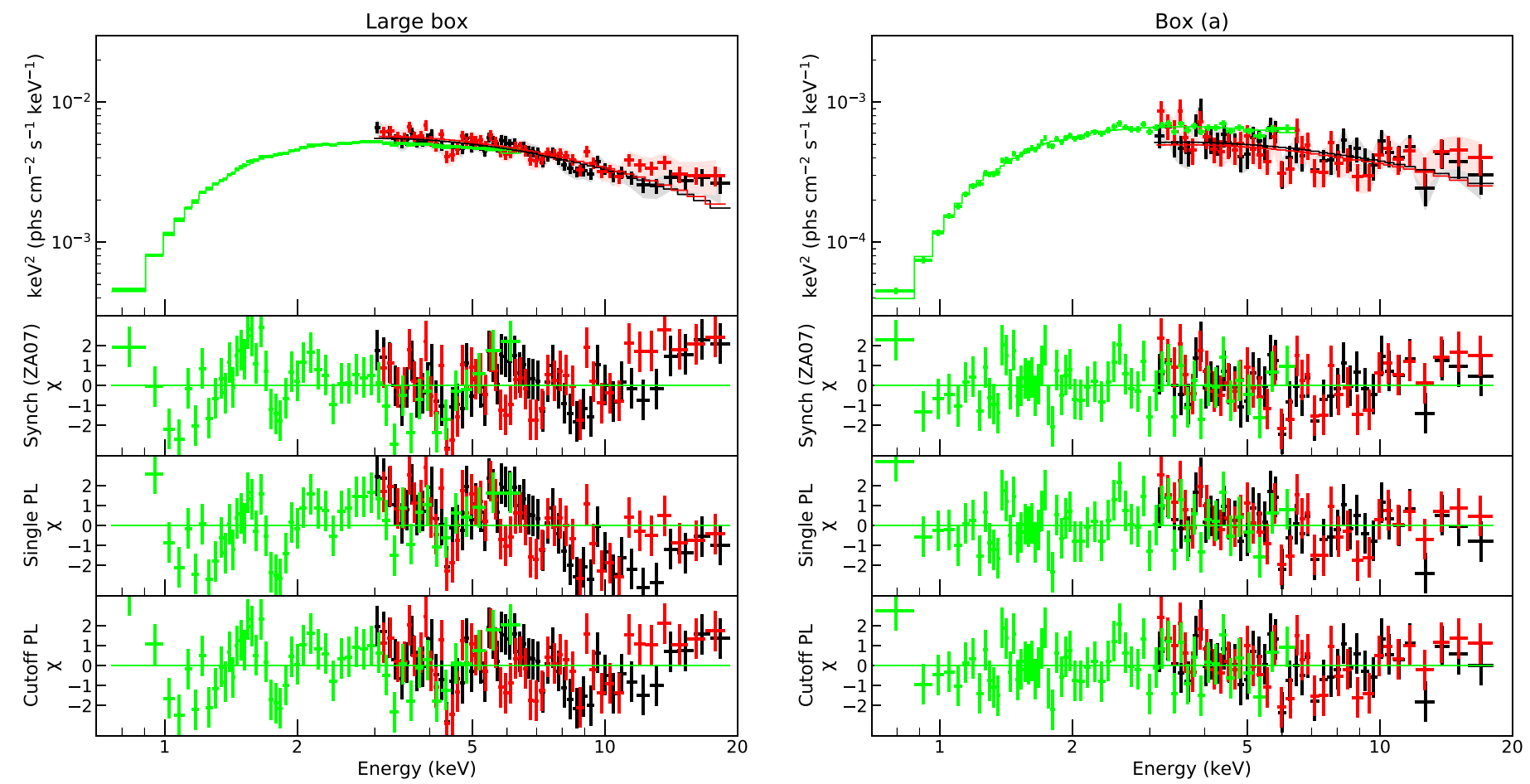

Figure 4. Spectrum of large box (left) and box (a) (right). Black, red, and green plots, respectively, show the spectrum by FPMA, FPMB, and Chandra. The top panel is the spectrum with the best-fit ZA07 model. The shadows represent the systematic uncertainties. The residuals from ZA07 model, single power-law model, and cutoff power-law model are shown in order from the second panel to the bottom, respectively.

filamentary structure of the SNR rim just behind the shock wave, $B$ is approximately $100 \mu \mathrm{G}$ in these subparsec regions, 0.01-0.4 pc (Bamba et al. 2003; Berezhko \& Völk 2006). The magnetic field is expected to be more enhanced, $B \sim 1 \mathrm{mG}$, in the smaller $(0.05 \mathrm{pc})$ region, derived from year-scale flux variation (Uchiyama et al. 2007; Uchiyama \& Aharonian 2008). The box (b), (c), (e), and (f) can be different from these filamentary and small structures in terms of the location and the size. The filament-like structure at box (c) and the inner edgelike structure between boxes (e) and (f) are, respectively, 1.8 and 2.6 pc away from the forward shock at box (a). The size is about $0.4-1 \mathrm{pc}$. We might need another scenario for the magnetic field enhancement in these comparably large subparsec regions that are isolated from the shock front.

The particle acceleration at reverse shock and/or reflection shock can be feasible for the observed higher cutoff energy parameters and the slower velocities. Given the position and the speed, the reverse shock does not seem to represent the filament and the inner edge from the hydrodynamical point of view, although it is not completely excluded (Tsuji \& Uchiyama 2016). The reflection shock, resulting from the interaction between the SNR shock and the ambient molecular cloud, is reasonable in the case of RX J1713.7-3946 NW, as discussed in Okuno et al. (2018). The evidence of the molecular cloud and the dense clump has been reported (Fukui et al. 2003, 2012; Sano et al. 2015). The shock-cloud interaction causes the deceleration of the shock, which is consistent with the measured slow speeds, and the magnetic field amplification (Inoue et al. 2012). However, it is not enough to consider only the standard acceleration at the reflection shock. If the reflection shock exists at the filament or the inner edge, it encounters the ejecta that is freely expanding outward. In the rest frame of the reflection shock, the upstream velocity is given by

$$
u_{1}=\frac{R_{\mathrm{ref}}}{t_{\mathrm{age}}}-v_{\mathrm{obs}},
$$

where $R_{\text {ref }}, t_{\mathrm{age}}$, and $v_{\mathrm{obs}}$ are the position of the reflection shock, the SNR age, and the observed apparent velocity, respectively (Sato et al. 2018). Assuming SN 393 is the supernova that created RX J1713.7-3946 ( $\left.t_{\mathrm{age}} \approx 1600 \mathrm{yr}\right), u_{1}$ is estimated to be $\sim 2700 \mathrm{~km} \mathrm{~s}^{-1}$ at box (c) and $\sim 2900 \mathrm{~km} \mathrm{~s}^{-1}$ at box (e). Using the obtained $u_{1}$ as the shock speed in Equation (3), the observed cutoff energy parameter still appears slightly larger than the theoretical value. This also suggests that the standard picture of DSA and the synchrotron cooling is not the case.

The presence of the magnetic turbulence can considerably affect the spectral shape of the synchrotron radiation (Bykov et al. 2008; Zirakashvili \& Aharonian 2010; Kelner et al. 2014). The turbulence can be described by different physical quantities, for example, with the power spectrum or with the correlation function.

In the case of large-scale turbulence, i.e., with the dissipation scale exceeding the photon formation length, the probability distribution of the magnetic field determines the emission spectrum (e.g., Kelner et al. 2014). Derivation of this distribution function from the first principles is a complicated task and is beyond the scope of our paper. Zirakashvili \& Aharonian (2010), however, have obtained an analytical form of the radiation spectrum in the presence of this distribution for the case of turbulence induced by the Weibel instability: the cutoff shape of the synchrotron radiation is described by $\exp \left(-\varepsilon^{1 / 3}\right)$ rather than $\exp \left(-\varepsilon^{1 / 2}\right)$ (see the appendix in Zirakashvili \& Aharonian 2010). Since this seems to be a feasible scenario for SNR shocks, we also utilize their model, resulting in both the obtained cutoff energy parameter and the 
Table 2

The Best-fit Parameters of the Spectral Analysis

\begin{tabular}{|c|c|c|c|c|c|c|c|}
\hline Box & Model & $\begin{array}{c}N_{\mathrm{H}} \\
\left(10^{22} \mathrm{~cm}^{-2}\right)\end{array}$ & $\Gamma$ & $\begin{array}{l}\varepsilon_{c} / \varepsilon_{0} \\
(\mathrm{keV})\end{array}$ & $\begin{array}{c}\text { Flux }_{3-20 \mathrm{kev}^{\mathrm{a}}} \\
\left(10^{-12} \mathrm{erg} \mathrm{cm}^{-2} \mathrm{~s}^{-1}\right)\end{array}$ & $\chi^{2}$ & (dof) \\
\hline $\operatorname{Large}^{b}$ & PL & $0.815 \pm 0.011$ & $2.32 \pm 0.02$ & $\ldots$ & $13.4 \pm 0.1$ & 92.9 & 48 \\
\hline $\operatorname{Large}^{c}$ & PL & $0.815 ;$ fixed & $2.55 \pm 0.04 \pm 0.02$ & $\cdots$ & $12.1_{-0.3}^{+0.2} \pm 0.4$ & 136 & 100 \\
\hline Large & PL & $0.836 \pm 0.011 \pm 0.001$ & $2.36 \pm 0.02 \pm 0.001$ & $\ldots$ & $12.4 \pm 0.2 \pm 0.4$ & 338 & 149 \\
\hline Large & CPL & $0.782 \pm 0.014 \pm 0.005$ & $2.15 \pm 0.04 \pm 0.02$ & $18.8_{-3.0_{-2.1}^{+4.2}}^{+2.6}$ & $11.9_{-0.3}^{+0.2} \pm 0.4$ & 248 & 148 \\
\hline Large & CPL & $0.740 \pm 0.007 \pm 0.002$ & $2 ;$ fixed & $11.4 \pm 0.5 \pm 0.2$ & $11.6_{-0.3}^{+0.2} \pm 0.4$ & 280 & 149 \\
\hline Large & ZA07 & $0.752 \pm 0.008 \pm 0.002$ & $\cdots$ & $1.14 \pm 0.06 \pm 0.02$ & $11.8_{-0.3}^{+0.2} \pm 0.4$ & 251 & 149 \\
\hline box (a) & ZA07 & $0.754 \pm 0.020 \pm 0.001$ & $\ldots$ & $1.90_{-0.32}^{+0.45} \pm 0.01$ & $1.26_{-0.13}^{+0.05} \pm 0.02$ & 151 & 136 \\
\hline box (b) & ZA07 & $0.775 \pm 0.023 \pm 0.001$ & $\cdots$ & $0.923_{-0.110}^{+0.136}{ }_{-0.008}^{+0.009}$ & $1.02_{-0.10}^{+0.05} \pm 0.03$ & 164 & 120 \\
\hline box (c) & ZA07 & $0.825_{-0.020}^{+0.021} \pm 0.001$ & $\cdots$ & $1.03_{-0.11}^{+0.14} \pm 0.01$ & $1.45_{-0.09}^{+0.05} \pm 0.04$ & 151 & 150 \\
\hline box (d) & ZA07 & $0.761_{-0.019}^{+0.020} \pm 0.001$ & $\cdots$ & $1.26_{-0.16}^{+0.20} \pm 0.01$ & $1.02_{-0.09}^{+0.05} \pm 0.02$ & 167 & 141 \\
\hline box (e) & ZA07 & $0.663 \pm 0.021 \pm 0.001$ & $\cdots$ & $1.44_{-0.23}^{+0.31} \pm 0.02$ & $1.14_{-0.12}^{+0.06} \pm 0.04$ & 121 & 113 \\
\hline box (f) & ZA07 & $0.490_{-0.021}^{+0.022} \pm 0.005$ & $\cdots$ & $0.598_{-0.075}^{+0.093}{ }_{-0.025}^{+0.030}$ & $1.55_{-0.18}^{+0.10} \pm 0.16$ & 191 & 131 \\
\hline
\end{tabular}

Notes. The errors refer to the $90 \%$ confidence level. The first and second errors of the flux indicate the statistic and the systematic uncertainties, respectively.

${ }^{a}$ Flux is calculated by using FPMA. The difference between FPMA and FPMB is smaller than the statistical error.

${ }^{\mathrm{b}}$ Using only Chandra data. Flux is calculated in $0.5-7 \mathrm{keV}$.

${ }^{\mathrm{c}}$ Using only NuSTAR data. $N_{\mathrm{H}}$ is fixed to the value obtained from the Chandra spectrum.

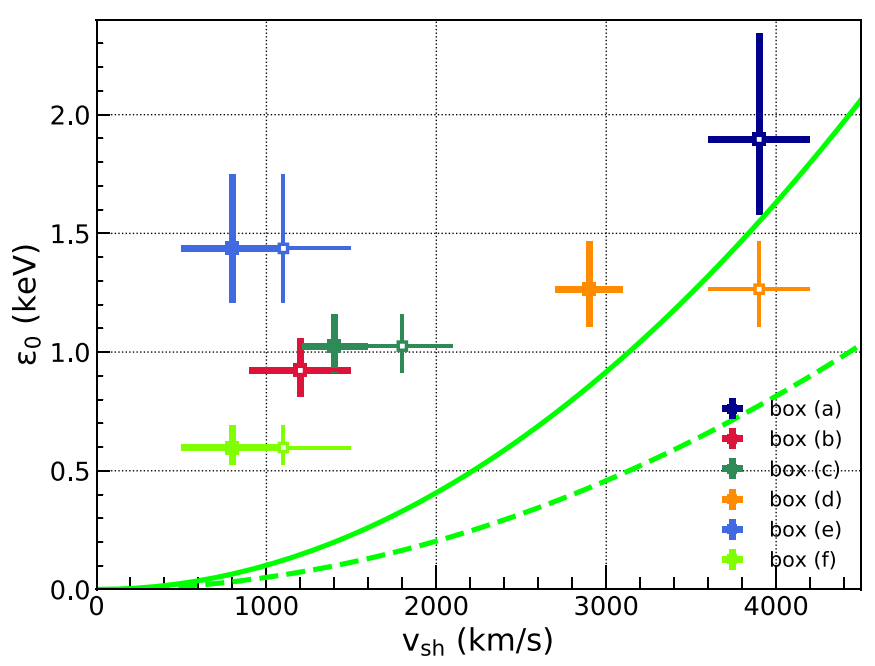

Figure 5. Relation between shock velocity and cutoff energy parameter. The error of the cutoff energy parameter shown here is only statistical. Note that the systematic error is much smaller. The green lines indicate the theoretical relation (Equation (3)) proposed in Zirakashvili \& Aharonian (2007). Solid and dashed lines are the cases of $\eta=1$ (Bohm limit) and $\eta=2$, respectively. Open markers show the projection-corrected velocities for boxes (c)-(f) assuming a spherical shell with the radius of $30^{\prime}$, i.e., the position of box (a).

theoretical prediction being about one order of magnitude smaller than those in the nonturbulent (monochromatic) field. This eventually produces almost the same trend shown in Figure 5, where boxes (a) and (d) are well described with the theoretical curve, and the rest are not.

On the other hand, there is a general relation that describes the impact of turbulence on the diffusion coefficient. We utilize this relation, which links the power spectrum slope and the energy dependence of the diffusion coefficient, to obtain two characteristic energy-dependences of the diffusion coefficient expected in the case of hydrodynamic and MHD turbulence. The analytical expressions, Equations (1)-(3), are derived in the case of Bohm diffusion, where the diffusion coefficient is proportional to the energy of particle, i.e., $D(E) \propto E^{\alpha}$ with $\alpha=1$. In this case, the electron spectrum has the exponential cutoff form of $\exp \left(-E^{2}\right)$ and the corresponding synchrotron spectrum has $\exp (-\sqrt{\varepsilon})$. If the diffusion coefficient deviates from the Bohm diffusion, i.e., $\alpha \neq 1$, the cutoff shape becomes somewhat different. We can diagnose the diffusion type from the determination of the cutoff shape (Zirakashvili \& Aharonian 2007; Yamazaki et al. 2014). This is beyond the scope of this paper and will be discussed in future publications.

\section{Conclusions}

We present the first result of the hard X-ray observations of the NW rim of SNR RX J1713.7-3946 with NuSTAR. The observations are heavily contaminated by the stray lights from nearby X-ray sources and the nonuniform stray-light contribution of CXB and GRXE. Using nuskybgd and adding the component of GRXE, we carefully estimate the complex background. For the first time, the spatially resolved nonthermal emission up to $20 \mathrm{keV}$ is detected from the remnant. The morphology taken with NUSTAR is broadly similar to the soft image in the previous work, except for HXC that is observed in the $10-20 \mathrm{keV}$ image of P1 epoch. We investigate the spectral distribution in the NW shell with the analytical expression of the synchrotron radiation in the vicinity of the SNR shock. The obtained cutoff energy is somewhat variable, from 0.6 to $1.9 \mathrm{keV}$. The particle acceleration is required to proceed in the regime close to Bohm limit near the forward shock. On the other hand, we need another scenario to explain the higher cutoff energy than theoretically predicted in the regions with slow speeds, such as the inner edge and the filamentary structure. The parsec-scale amplification of the magnetic field and/or the acceleration at the reflection shock might be the case.

We thank Kaya Mori and Daniel R. Wik for the helpful advice about NuSTAR analysis, and Dmitry Khangulyan for a fruitful discussion. We also thank the anonymous referee for the useful comments. This work has made use of data from the NuSTAR mission, a project led by the California Institute of Technology, managed by the Jet Propulsion Laboratory, and funded by the National Aeronautics and Space Administration. 
This work is supported by the NUSTAR Cycle 1 observation program. We appreciate the NUSTAR Operations, Software, and Calibration teams for support with the execution and analysis of these observations. N.T. is supported by the Japan Society for the Promotion of Science (JSPS) KAKENHI grant No. JP17J06025. R.K. acknowledges support from the Russian Science Foundation (grant 19-12-00369). This work was partially supported by JSPS KAKENHI grant Nos. JP18H03722 and JP18H05463.

Software: NuSTARDAS (v1.4.1), HEAsoft (v6.19), nuskybgd (https://github.com/NuSTAR/nuskybgd; Wik et al. 2014), XSPEC (v12.9.0, Arnaud 1996), CIAO (v4.9, Fruscione et al. 2006).

\section{ORCID iDs}

Naomi Tsuji (1) https://orcid.org/0000-0001-7209-9204

David Berge (1) https://orcid.org/0000-0002-2918-1824

Roman Krivonos (1) https://orcid.org/0000-0003-2737-5673

Takaaki Tanaka @ ittps://orcid.org/0000-0002-4383-0368

\section{References}

Abdo, A. A., Ackermann, M., Ajello, M., et al. 2011, ApJ, 734, 28 Acero, F., Katsuda, S., Ballet, J., \& Petre, R. 2017, A\&A, 597, A106 Aharonian, F., Sun, X.-n., \& Yang, R.-z. 2017, A\&A, 603, A7

Arnaud, K. A. 1996, in ASP Conf. Ser. 101, Astronomical Data Analysis Software and Systems V, ed. G. H. Jacoby \& J. Barnes (San Francisco, CA: ASP), 17

Bamba, A., Yamazaki, R., Ueno, M., \& Koyama, K. 2003, ApJ, 589, 827

Berezhko, E. G., \& Völk, H. J. 2006, A\&A, 451, 981

Boldt, E. 1987, in IAU Symp. 124, Observational Cosmology, ed. A. Hewitt, G. Burbidge, \& Z.-F. Li (Dordrecht: D. Reidel), 611
Bykov, A. M., Uvarov, Y. A., \& Ellison, D. C. 2008, ApJL, 689, L133

Fesen, R. A., Kremer, R., Patnaude, D., \& Milisavljevic, D. 2012, AJ, 143, 27

Fruscione, A., McDowell, J. C., Allen, G. E., et al. 2006, Proc. SPIE, 6270, $62701 \mathrm{~V}$

Fukui, Y., Moriguchi, Y., Tamura, K., et al. 2003, PASJ, 55, L61

Fukui, Y., Sano, H., Sato, J., et al. 2012, ApJ, 746, 82

Grefenstette, B. W., Reynolds, S. P., Harrison, F. A., et al. 2015, ApJ, 802, 15

Harrison, F. A., Craig, W. W., Christensen, F. E., et al. 2013, ApJ, 770, 103

H.E.S.S. Collaboration, Abdalla, H., Abramowski, A., et al. 2018, A\&A, 612, A6

Inoue, T., Yamazaki, R., Inutsuka, S., \& Fukui, Y. 2012, ApJ, 744, 71

Katsuda, S., Acero, F., Tominaga, N., et al. 2015, ApJ, 814, 29

Kelner, S. R., Lefa, E., Rieger, F. M., \& Aharonian, F. A. 2014, ApJ, 785, 141

Krivonos, R., Revnivtsev, M., Churazov, E., et al. 2007b, A\&A, 463, 957

Krivonos, R., Revnivtsev, M., Lutovinov, A., et al. 2007a, A\&A, 475, 775

Li, J.-T., Ballet, J., Miceli, M., et al. 2018, ApJ, 864, 85

Lopez, L. A., Grefenstette, B. W., Reynolds, S. P., et al. 2015, ApJ, 814, 132

Moretti, A., Pagani, C., Cusumano, G., et al. 2009, A\&A, 493, 501

Okuno, T., Tanaka, T., Uchida, H., Matsumura, H., \& Tsuru, T. G. 2018, PASJ, 70, 77

Revnivtsev, M., Sazonov, S., Gilfanov, M., Churazov, E., \& Sunyaev, R. 2006, A\&A, 452, 169

Sano, H., Fukuda, T., Yoshiike, S., et al. 2015, ApJ, 799, 175

Sato, T., Katsuda, S., Morii, M., et al. 2018, ApJ, 853, 46

Tanaka, T., Uchiyama, Y., Aharonian, F. A., et al. 2008, ApJ, 685, 988

Tsuji, N., \& Uchiyama, Y. 2016, PASJ, 68, 108

Uchiyama, Y., \& Aharonian, F. A. 2008, ApJL, 677, L105

Uchiyama, Y., Aharonian, F. A., Tanaka, T., Takahashi, T., \& Maeda, Y. 2007, Natur, 449, 576

Wang, Z. R., Qu, Q.-Y., \& Chen, Y. 1997, A\&A, 318, L59

Wik, D. R., Hornstrup, A., Molendi, S., et al. 2014, ApJ, 792, 48

Yamazaki, R., Ohira, Y., Sawada, M., \& Bamba, A. 2014, RAA, 14, 165

Yuasa, T., Makishima, K., \& Nakazawa, K. 2012, ApJ, 753, 129

Zirakashvili, V. N., \& Aharonian, F. 2007, A\&A, 465, 695

Zirakashvili, V. N., \& Aharonian, F. A. 2010, ApJ, 708, 965

Zoglauer, A., Reynolds, S. P., An, H., et al. 2015, ApJ, 798, 98 